\title{
Clinical Characteristics, Surgical Management and Adjuvant Therapy of Patients with Uterine Carcinosarcoma: A Retrospective Case Series
}

\author{
Klinische Charakteristika, operatives Management und adjuvante Therapie \\ von Patientinnen mit uterinen Karzinosarkomen: eine retrospektive Fallserie
}

Authors

Affiliations
C. Wallwiener ${ }^{1}$, A. Hartkopf ${ }^{1}$, S. Kommoss ${ }^{1}$, C. Joachim ${ }^{1}$, M. Wallwiener ${ }^{2}$, F. A. Taran ${ }^{1}$, S. Brucker ${ }^{1}$

${ }^{1}$ Department of Obstetrics and Gynecology, University of Tübingen, Tübingen

${ }^{2}$ Department of Obstetrics and Gynecology, University of Heidelberg, Heidelberg

\author{
Key words \\ - uterine carcinosarcoma \\ - uterine neoplasms \\ - surgical staging \\ - adjuvant therapy \\ Schlüsselwörter \\ - uterines Karzinosarkom \\ - uterine Neoplasien \\ - chirurgisches Staging \\ - adjuvante Therapie
}

received 24.8.2015

revised 28.12.2015

accepted 29.12.2015

\section{Bibliography}

Dol http://dx.doi.org/

10.1055/s-0042-100205

Geburtsh Frauenheilk 2016; 76:

188-193 @ Georg Thieme

Verlag KG Stuttgart · New York . ISSN 0016-5751

\section{Correspondence}

Florin-Andrei Taran, MD

Department of Obstetrics

and Gynecology

University of Tübingen

Calwerstraße 7

72076 Tübingen

florin-andrei.taran@

med.uni-tuebingen.de

\section{Abstract \\ $\nabla$}

Purpose: To review a single-center experience over a 27-year period of managing uterine carcinosarcoma (UCS), focusing on surgical practice, adjuvant therapy and clinical outcome.

Material and Methods: This was a retrospective study of women with histologically proven UCS treated at the Department of Obstetrics and Gynecology, University of Tübingen, Germany, between 1983 and 2010. Inpatient and outpatient records were reviewed; follow-up and survival data were ascertained.

Results: The study population comprised 18 patients with UCS. Primary surgical treatment consisted of total abdominal hysterectomy in 12 patients (67\%) and laparoscopic total hysterectomy in 4 patients (22\%). Bilateral salpingo-oophorectomy was performed in $94 \%$ of patients (17/18). Lymph nodes were evaluated in 15 patients (83\%). Positive pelvic lymph nodes were present in 2 patients (11\%). A total of 17 patients (94\%) received adjuvant therapy. Disease recurred in 7 (39\%) patients of our study group, with no recurrence noted in the 4 patients who underwent laparoscopic surgical staging. Median disease-free survival (DFS) was 48.7 months (95\% CI: $0.0-$ 157.3) and median overall survival (OS) was 49.9 months (95\% CI: 0.0-108.2). The 5-year survival rate was $40 \%$.

Conclusion: UCS is a rare and aggressive uterine neoplasm with high recurrence rates and metastatic potential. Surgical staging consisting of total hysterectomy with bilateral salpingo-oophorectomy and systematic lymphadenectomy is the most important treatment for patients with UCS. Adjuvant radiation therapy appears to decrease pelvic recurrence, but there is a high incidence of distant recurrence, indicating the need for additional systemic treatment.

\section{Zusammenfassung \\ $\nabla$}

Einleitung: Im Folgenden wird die Erfahrung eines Zentrums aus 27 Jahren bezüglich des Managements von uterinen Karzinosarkomen (UKS) mit besonderem Fokus auf operative Techniken, adjuvante Therapie und klinisches Outcome dargestellt.

Material und Methoden: Dies ist eine retrospektive Studie von Frauen mit einem histologischen Nachweis eines UKS, die in der Universitätsfrauenklinik Tübingen zwischen den Jahren 1983 und 2010 therapiert wurden. Sowohl der stationäre Aufenthalt als auch die ambulante Betreuung wurden analysiert, die Überlebensdaten wurden exploriert.

Ergebnisse: Die Studienpopulation beinhaltete 18 Patientinnen mit UKS. Die operative Primärtherapie beinhaltete entweder eine totale abdominale Hysterektomie bei 12 Patientinnen (67\%), oder eine totale laparoskopische Hysterektomie bei 4 Patientinnen (22\%). Eine systematische Lymphonodektomie wurde bei 15 Patientinnen (83\%) durchgeführt, eine Nodalpositivität ergab sich bei 2 Patientinnen (11\%). In Summe erhielten 17 Frauen (94\%) eine adjuvante Therapie. Ein Rezidiv zeigte sich bei insgesamt 7 Patientinnen (39\%), keine der 4 Patientinnen nach minimalinvasiver operativer Therapie hatte ein Rezidiv. Das mediane krankheitsfreie Überleben betrug 48,7 Monate, das mediane Gesamtüberleben betrug 49,9 Monate. Das 5-Jahres-Überleben betrug 40\%.

Zusammenfassung: UKS sind sehr seltene und aggressive uterine Neoplasien mit einem hohen Rezidiv- und Metastasierungsrisiko. Die Therapie beinhaltet als wichtigstes Element das chirurgische Staging bestehend aus totaler Hysterektomie mit beidseitiger Adnexektomie und systematischer Lymphonodektomie. Die adjuvante Radiotherapie senkt die Inzidenz von Beckenrezidiven, allerdings deutet die hohe Rate an Fernmetastasen auf die Vorteile einer systemischen Therapie hin. 


\section{Introduction}

\section{$\nabla$}

Uterine carcinosarcoma (UCS), also known as malignant mixed mesodermal tumor or malignant mixed mullerian tumors (MMMT), is an extremely rare and very aggressive uterine neoplasm. UCS accounts for less than 5\% of all uterine malignancies but is responsible for $15 \%$ of all deaths caused by uterine malignancies [1]. UCS is composed of both epithelial and mesenchymal elements [1]. UCS was previously considered to behave like a sarcoma, but recent molecular and genetic data have suggested that the epithelial component of the UCS is the primary driver of tumor aggressiveness and these rare neoplasms are now grouped with endometrial carcinoma [1]. This has led to a revision in sarcoma staging, with leiomyosarcoma and endometrial stromal tumor assigned a separate and distinct staging system and UCS grouped with endometrial carcinomas [2].

UCS usually develops in postmenopausal women; commonly reported symptoms of UCS include abnormal uterine bleeding, bloody/watery vaginal discharge, abdominal pain, and the presence of a pelvic mass [3]. Known epidemiological risk factors for the development of UCS are increased estrogen levels (especially exogenous exposure), obesity, nulliparity, and ethnicity [3]. Black women have a higher risk of developing UCS and lower survival rates compared to white women [4]. Prolonged tamoxifen use and a history of pelvic irradiation have also been associated with the development of UCS [1,5-7].

The initial treatment recommended for early-stage UCS is surgical staging, defined as hysterectomy, bilateral salpingo-oophorectomy and systematic lymphadenectomy. In patients with newly diagnosed advanced-stage UCS, cytoreductive surgery with complete gross resection was found to improve overall survival (OS) [8]. Data on adjuvant chemotherapy and/or radiation therapy for UCS are limited and often conflicting. Recently, the German Society of Obstetrics and Gynecology issued an evidence-based guideline on the appropriate management of these heterogeneous tumors [9]. The 2010 National Comprehensive Cancer Network (NCCN) guidelines recommend adjuvant treatment for all stages of UCS, except for stage IA disease [10]. The optimal chemotherapeutic regimen is, however, still debated. Adjuvant pelvic radiation therapy has been shown to decrease the risk of pelvic recurrence and delay metastasis; however, the increased rate of distant recurrence associated with disease progression underlines the need for systemic therapy [1].

Given the rarity of UCS and of uterine sarcomas in general, attempts to conduct prospective trials in order to improve therapy regimens and develop disease-specific (surgical and adjuvant) and individualized management strategies have been challenging. Accordingly, in the absence of prospective data, there is a continued need to review past practice. Thus, we previously published our experience of managing uterine leiomyosarcoma and endometrial stromal tumors [11,12]. The aim of the present study was to review our experience in the management of UCS, focusing on surgical and adjuvant treatment strategies and clinical outcomes.

\section{Material and Methods}

\section{$\nabla$}

Study design and identification of the patient cohort This was a retrospective study conducted in the Department of Obstetrics and Gynecology of the University of Tübingen, Germany. Using the institutional databases of the clinical cancer registry of the Comprehensive Cancer Center (CCC) Tübingen, we identified all women included in the registry between January 1st, 1983 and January 31st, 2010 with a diagnosis of either "UCS", "malignant mixed mesodermal tumor" or "malignant mixed mullerian tumor (MMMT)" as their final diagnosis.

Diagnoses of the identified UCS cases were compared manually with the pathology reports; the sources agreed in 18 of 19 (95\%) cases. Only histologically confirmed cases were analyzed. One case was excluded because histology resulted in a diagnosis of leiomyosarcoma of the uterus. Thus, the study group comprised 18 patients with a diagnosis of UCS.

A retrospective review of both inpatient and outpatient medical records (from the Department of Obstetrics and Gynecology, University of Tübingen) was performed to identify sociodemographic and anthropometric variables and to confirm intraoperative and pathology findings. Data on adjuvant treatment was recorded. The clinical cancer registry of the CCC Tübingen provided the data on follow-up and survival. Time to disease recurrence and death or time to last contact were calculated. Premenopausal status was defined as the occurrence of at least one menstrual period within 12 months before surgery. Adjuvant treatment was administered to selected patients based on decisions taken by the tumor board. We assessed the disease stage retrospectively for every patient using the 2009 International Federation of Gynecology and Obstetrics (FIGO) staging system [13].

\section{Statistical analysis}

Data were coded and entered into an Excel spreadsheet version 2007 (Microsoft, Redmond, Washington DC, USA). Statistical analysis was carried out using JMP for Windows version 8.0 (SAS Institute Cary, NC, USA) and Prism 5 (GraphPad Software, La Jolla, CA, USA). Means and standard deviations (SD) are reported for continuous variables and frequency counts and percentages for categorical variables. Survival curves were generated using the Kaplan-Meier method. Calculation of disease-free survival (DFS): no follow-up data was available for 9 patients; 2 patients had no disease recurrence at last follow-up and 7 patients had disease recurrence. Calculation of overall survival: no followup data was available for 7 patients; 3 patients were recorded as alive at last follow-up and 8 patients had died of UCS.

\section{Results}

$\nabla$

\section{Characteristics of the study cohort}

The study sample comprised 18 patients with UCS who underwent treatment at our institution. A total of 9 cases treated between January 1st, 1983 and December 31st, 1999 were identified; a further 9 women who underwent treatment for UCS between January 1st, 2000 and January 31st, 2010 were identified. The median follow-up for survivors was 115.7 months. Patient characteristics are summarized in Table 1 . The mean age was 65.0 years (range $48-83$ ). At the time of primary surgery, $83 \%$ of the patients were postmenopausal. Abnormal uterine bleeding was the most common presenting symptom and was reported in $56 \%$ of the patients. $11 \%$ of patients reported abdominal pain, a pelvic mass was described in $28 \%$ of the patients, and $28 \%$ of the patients reported both abdominal pain and abnormal bleeding as presenting symptoms ( $\bullet$ Table 1 ).

There were 10 patients (56\%) with FIGO stage I, 2 patients (11\%) with stage II, 2 patients (11\%) with stage III, and 4 patients $(22 \%)$ with stage IV disease. Distribution by grade showed 4 patients 
(22\%) with grade 1 disease, 3 patients (17\%) with grade 2 disease, 10 patients (56\%) with grade 3 disease, and one patient with grade 4 disease (6\%). Lymphovascular invasion was present in 4 $(22 \%)$ cases. Tumor markers were evaluated in 14 women and were found to be elevated in 4 women (22\%). CA 125 was elevated in 3 patients and CEA and CA 19-9 in one patient (data not shown).

\section{Surgery and adjuvant treatment}

Primary surgical treatment consisted of total abdominal hysterectomy in 12 patients (67\%) and laparoscopic total hysterectomy in 4 patients (22\%). Bilateral salpingo-oophorectomy (BSO) was performed in $94 \%$ of patients (17/18). Lymph nodes were evaluated in 15 patients (83\%); 6 patients underwent pelvic lymphadenectomy and 9 patients underwent pelvic and para-aortic lymphadenectomy ( $\bigcirc$ Table 1 ). Positive pelvic lymph nodes were present in 2 patients (11\%); one patient (6\%) had positive pelvic and para-aortic lymph nodes.

A total of 17 patients (94\%) received adjuvant therapy ( $\bullet$ Table 1 ). Of those patients, 2 patients received adjuvant chemotherapy. Adjuvant radiation therapy was administered to 8 patients (44\%) and 7 patients (39\%) received combined adjuvant radiation therapy and chemotherapy ( $\bullet$ Table $\mathbf{1}$ ). Chemotherapy regimens consisted of ifosfamide, doxorubicin, non-pegylated liposomal doxorubicin, cisplatin, carboplatin, gemcitabine and paclitaxel; the administered chemotherapy regimen was not on record for 3 patients.

\section{Disease recurrence and survival analysis}

Seven (39\%) patients in our study group had disease recurrence. No trocar recurrence occurred in the 4 patients who underwent laparoscopic surgical staging. The median follow-up for the two survivors who underwent laparoscopic surgery was 67.7 months. Three women had pelvic recurrence, 3 women had distant recurrence, and 1 patient had both pelvic and distant recurrence. The sites of recurrence included the vagina, pelvis, lungs, bone, retroperitoneum and brain. $\triangle$ Table 2 gives an overview of the patients with disease recurrence ( $\boldsymbol{O}$ Table 2 ).

Disease-free survival (DFS) and overall survival (OS) of patients with UCS are shown in 0 Figs. 1 and 2. Median DFS in our study group was 48.7 months (95\% CI: 0.0-157.3), and median OS was 49.9 months (95\% CI: 0.0-108.2) (O Fig. 1 and Fig. 2, respectively). The 5 -year survival rate was $40 \%$ for all patients.

\section{Discussion}

$\nabla$

UCS are a rare and very aggressive subtype of uterine malignancy. Due to the rarity of the disease, there are no high-level evidencebased guidelines on the therapeutic management of this entity.
Table 1 Characteristics of the 18 patients with carcinosarcoma of the uterus.

\begin{tabular}{|c|c|}
\hline \multirow[t]{2}{*}{ Characteristic } & Value \\
\hline & Mean \\
\hline Age at diagnosis, years (range; \pm SD) & $65.0(48-83 ; 11.8)$ \\
\hline Size of uterine lesion $( \pm \mathrm{SD}), \mathrm{cm}$ & $7.1(4.7)$ \\
\hline Uterine/composite compound weight ( \pm SD), g & $218.4(150.7)$ \\
\hline Menopausal status & No. (\%) \\
\hline D Premenopausal & $3(16.7)$ \\
\hline Postmenopausal & $15(83.3)$ \\
\hline \multicolumn{2}{|l|}{ Preoperative symptoms } \\
\hline - Pelvic mass & $5(27.8)$ \\
\hline Abdominal pain & $2(11.1)$ \\
\hline Abnormal bleeding & $10(55.6)$ \\
\hline - Abdominal pain and abnormal bleeding & $5(27.8)$ \\
\hline \multicolumn{2}{|l|}{ FIGO stage } \\
\hline$>1$ & $10(55.6)$ \\
\hline$>11$ & $2(11.1)$ \\
\hline - III & $2(11.1)$ \\
\hline D IV & $4(22.2)$ \\
\hline Surgical therapy & $18(100.0)$ \\
\hline - Total abdominal hysterectomy & $12(66.7)$ \\
\hline - Total laparoscopic hysterectomy & $4(22.2)$ \\
\hline Debulking surgery & $2(11.1)$ \\
\hline - Bilateral salpingo-oophorectomy & $17(94.4)$ \\
\hline Lymphadenectomy & $15(83.3)$ \\
\hline pelvic & $6(33.3)$ \\
\hline pelvic/para-aortic & $9(50.0)$ \\
\hline Adjuvant therapy & $17(94.4)$ \\
\hline - Adjuvant chemotherapy & $2(11.1)$ \\
\hline - Adjuvant radiation therapy & $8(44.4)$ \\
\hline - Combined adjuvant therapy & $7(38.9)$ \\
\hline
\end{tabular}

Hence, there is a need for individual centers to report even retrospective data on the surgical management, adjuvant treatment, and clinical outcome of patients with this disease. The present study reviewed the experience of our department in managing UCS over a period of three decades.

Our findings are consistent with previously reported five-year survival rates for UCS, which are reported to range from 33 to $39 \%[1,14]$. Interestingly, in our case series, the OS was not significantly longer compared to the DFS. A possible explanation for this rather paradoxical finding could be the fact that 2 patients died within 2 months of their initial UCS diagnosis; thus, these patients actually died of primary and not of recurrent disease.

The initial treatment recommended for UCS is surgical staging for early-stage disease, with cytoreduction proposed for advancedstage disease. Absence of primary surgical staging and/or incomplete cytoreduction have been shown to be independent prognostic factors for survival [8]. Because of the small numbers of

Table 2 Overview and treatment of patients with disease recurrence.

\begin{tabular}{|c|c|c|c|c|}
\hline Patient & Site of recurrence & Time to recurrence (months) & Treatment for disease recurrence & Time to death (months) \\
\hline 1 & brain & 3.6 & palliative & 0 \\
\hline 2 & bone, lung & 11.5 & palliative & 0 \\
\hline 3 & vagina & 54.0 & surgery/radiation therapy & 31.3 \\
\hline 4 & vagina & 9.5 & surgery/radiation therapy & 15.8 \\
\hline 5 & retroperitoneum & 48.7 & palliative & 1.3 \\
\hline 6 & lung, vagina & 8.4 & chemotherapy/radiation therapy & 27.0 \\
\hline 7 & pelvis & 123.0 & palliative & 0 \\
\hline
\end{tabular}




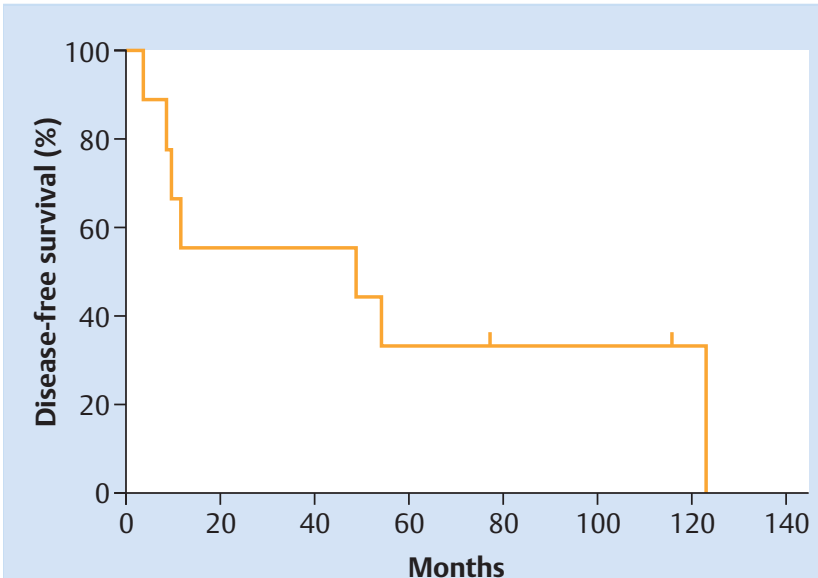

Fig. 1 Kaplan-Meier analysis of disease-free survival (DFS) of patients with carcinosarcoma of the uterus.

patients, the optimal approach for the surgical staging of UCS has not been the focus of recent scientific appraisal. Very few patients with UCS (41 of 2489 patients, representing 1.6\% of the study population) were eligible for inclusion in the Gynecologic Oncology Group (GOG) LAP2 study [15]. The authors of that study showed that comprehensive staging of uterine cancers can be performed laparoscopically with only a small difference in recurrence rates [15]. Three of four presumed trocar site recurrences occurred in patients with advanced disease [15]. Similarly, we observed no recurrence in 4 patients (22\%) of our study group with early-stage disease staged laparoscopically. Laparoscopic surgical staging can therefore be considered in selected patients with UCS [1]. Nevertheless, these findings on the laparoscopic staging of UCS have to be interpreted with caution due to the limited number of patients and warrant future investigation.

Lymph node metastasis is much more common in patients with UCS compared to patients with uterine leiomyosarcoma or patients with endometrial stromal tumor [14]. Thus, lymphadenectomy is mandatory for the management of patients with UCS to improve surgical staging and allow a better selection of patients for adjuvant treatment. The majority of patients (83\%) included in the present study underwent systematic lymphadenectomy. Nevertheless, the exact role and extent of lymphadenectomy in patients with UCS remains controversial.

Some studies have reported no survival benefit from systematic lymphadenectomy in patients with UCS. However, the largest retrospective study to date by Nemani et al. evaluated the effects of lymphadenectomy in patients with UCS using Surveillance, Epidemiology, and End Results (SEER) data and reported that lymphadenectomy led to a significant improvement in overall survival for patients with UCS compared to patients who did not have lymphadenectomy $[16,17]$. The survival advantage was maintained regardless of whether patients who did not undergo lymphadenectomy received adjuvant radiation therapy or not [16]. However, the number of total lymph nodes removed $(<12$ vs. $\geq 12$ ), irrespective of whether they were positive or negative, was not predictive for overall survival, calling into question the idea that improved survival is based on the therapeutic value of lymphadenectomy. Moreover, the data presented in the study did not provide information on adjuvant chemotherapy, thus removing a potential confounder from the data analysis [18].

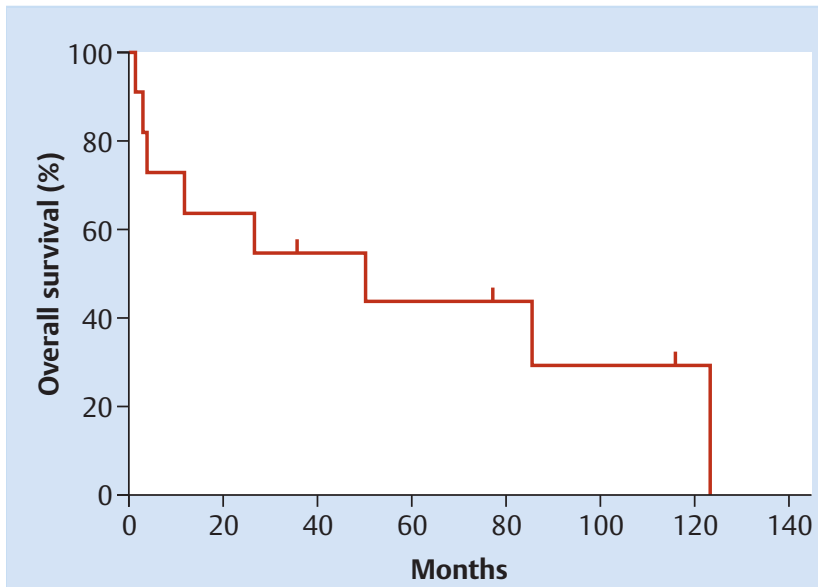

Fig. 2 Kaplan-Meier analysis of overall survival (OS) of patients with carcinosarcoma of the uterus.

In contrast, Temkin et al. found in a smaller retrospective study that the number of resected lymph nodes was the only risk factor correlated with recurrence and survival in patients with UCS [19]. They concluded that lymphadenectomy is crucial in patients with UCS to discover occult metastatic disease and potentially provide patients with a therapeutic benefit [19]. A new concept for the surgical staging of UCS was reported in a recent study by the Memorial Sloan Kettering Cancer Center, which suggested that progression-free survival in patients with UCS who undergo sentinel lymph node mapping with additional adjuvant therapy was similar to that of patients treated with systematic lymphadenectomy and additional adjuvant therapy [18]. However, given the fact that the experience with sentinel lymph node mapping, particularly for this disease, is limited and still at an early stage, additional prospective studies are needed to confirm the results and analyze the impact on overall survival for patients with UCS. The adjuvant therapy administered to the patients in our study consisted of different chemotherapy regimens and different adjuvant therapy approaches (radiation therapy alone versus combined chemotherapy/radiation therapy). This can be explained by the fact that patients were treated over a period which spanned 3 decades. But the results also highlight the ongoing debate about the optimal adjuvant therapy for women with UCS. In general, multimodal treatment is recommended and should be considered at all stages of disease because of the aggressive nature of UCS [1].

Initially, adjuvant chemotherapy strategies for UCS included only single agents, resulting in a wide range of response rates from 7$36 \%$, with ifosfamide showing the best response rate [3]. Subsequently, phase II and III studies looked at combination therapies with ifosfamide and reported a survival benefit $[1,3]$. A recent Cochrane review found that women with advanced-stage UCS who received combination chemotherapy which included ifosfamide had a lower risk of death and disease progression than women who received ifosfamide alone [20]. Nevertheless, given the high toxicity profile and complex administration schedule of ifosfamide the search for regimens with lower toxicity continues. The results of the GOG 261 study (NCT00954174), a randomized phase III trial of paclitaxel plus carboplatin versus ifosfamide plus paclitaxel in chemotherapy-naive patients with UCS, are eagerly awaited [21]. 
Several new targeted therapy compounds [aflibercept, AP23573 (mTOR inhibitor), imatinib, iniparib, pazobanib, sorafenib] have also been evaluated for the treatment of UCS, either as single agents or in combination chemotherapy regimens; however, the overall tumor response rates and achieved survival rates were poor [22-27].

Most studies on radiation therapy in patients with UCS report an improvement in pelvic control $[28,29]$. However, radiation therapy does not seem to confer a survival benefit [10]. A previous SEER data-based study by Wright et al. showed that radiation therapy reduced mortality rates in patients with UCS who had not undergone lymphadenectomy but had only a minimal effect on survival in node-negative women [30]. In contrast to this study, other studies have reported a survival benefit from lymphadenectomy alone but no survival benefit from radiation therapy with or without lymphadenectomy $[1,16]$. In the European Organisation for Research and Treatment of Cancer Gynaecological Cancer Group Study (protocol 55874), patients with uterine sarcoma stage I and II were randomized to either observation or pelvic radiation [30]. The study showed no difference in either overall or disease-free survival but reported increased local control for patients with UCS who received radiation therapy [31]. In conclusion, adjuvant radiation therapy appears to decrease pelvic recurrence in patients with UCS, but high rates of distant recurrence remain, indicating the need for additional systemic treatment [1].

The major limitations of our study are its retrospective design and the small number of patients with UCS. However, the study benefits from the long follow-up of patients and the assessment of their clinical characteristics. Prospective studies with larger cohorts and longer follow-up times could provide new prognostic factors to predict the prognosis of patients with UCS and improve our understanding of the pathogenesis of this very rare disease. Future studies should incorporate multiple institutions, address the role of sentinel lymph node mapping and systemic lymphadenectomy, consider patient selection criteria, and evaluate adjuvant therapy regimens (chemotherapy, radiation therapy) including targeted therapy compounds and the optimal sequencing of regimens to treat UCS.

\section{Conclusions for Practice}

\section{$\nabla$}

UCS is a rare and very aggressive subtype of uterine malignancy. The initial treatment recommended for early-stage UCS is surgical staging, defined as hysterectomy, bilateral salpingo-oophorectomy and systematic lymphadenectomy. Cytoreductive surgery with complete gross resection should be considered for newly diagnosed, advanced-stage UCS. Recommended adjuvant therapy consists of combination chemotherapy with or without radiation therapy and should be considered for all stages of disease because of the aggressive nature of UCS.

\section{Acknowledgement}

$\nabla$

We would like to Brigit Trilling for her assistance in obtaining patient follow-up data.

\section{Conflict of Interest}

None.

\section{References}

1 Cantrell LA, Blank SV, Duska LR. Uterine carcinosarcoma: a review of the literature. Gynecol Oncol 2015; 137: 581-588

2 Pradhan TS, Stevens EE, Ablavsky $M$ et al. FIGO staging for carcinosarcoma: can the revised staging system predict overall survival? Gynecol Oncol 2011; 123: 221-224

3 Artioli G, Wabersich J, Ludwig K et al. Rare uterine cancer: carcinosarcomas. Review from histology to treatment. Crit Rev Oncol Hematol 2015; 94: 98-104

4 Erickson BK, Doo DW, Zhang B et al. Black race independently predicts worse survival in uterine carcinosarcoma. Gynecol Oncol 2014; 133: 238-241

5 McCluggage WG, McManus DT, Lioe TF et al. Uterine carcinosarcoma in association with tamoxifen therapy. Br J Obstet Gynaecol 1997; 104: 748-750

6 Kloos I, Delaloge S, Pautier P et al. Tamoxifen-related uterine carcinosarcomas occur under/after prolonged treatment: report of five cases and review of the literature. Int J Gynecol Cancer 2002; 12: 496-500

7 Pothuri B, Ramondetta L, Eifel P et al. Radiation-associated endometrial cancers are prognostically unfavorable tumors: a clinicopathologic comparison with 527 sporadic endometrial cancers. Gynecol Oncol 2006; 103: 948-951

8 Tanner EJ, Leitao MM jr., Garg K et al. The role of cytoreductive surgery for newly diagnosed advanced-stage uterine carcinosarcoma. Gynecol Oncol 2011; 123: 548-552

9 Denschlag D, Thiel FC, Ackermann S et al. Sarcoma of the uterus. Guideline of the DGGG (S2k-Level, AWMF Registry No.015/074, August 2015). Geburtsh Frauenheilk 2015; 75: 1028-1042

10 Cantrell LA, Havrilesky L, Moore DT et al. A multi-institutional cohort study of adjuvant therapy in stage I-II uterine carcinosarcoma. Gynecol Oncol 2012; 127: 22-26

11 Rothmund R, Huebner M, Joachim C et al. Clinical characteristics, surgical management and adjuvant therapy of patients with uterine leiomyosarcoma: 27 years of experience. Geburtsh Frauenheilk 2011; 71: 1085-1089

12 Rothmund R, Hartkopf A, Joachim C et al. Clinical characteristics, pathological reevaluation, surgical management and adjuvant therapy of patients with endometrial stromal tumors. Arch Gynecol Obstet 2014; 290: 1195-1200

13 Page BR, Pappas L, Cooke EW et al. Does the FIGO 2009 endometrial cancer staging system more accurately correlate with clinical outcome in different histologies? Revised staging, endometrial cancer, histology. Int J Gynecol Cancer 2012; 22: 593-598

14 Vorgias G, Fotiou S. The role of lymphadenectomy in uterine carcinosarcomas (malignant mixed mullerian tumours): a critical literature review. Arch Gynecol Obstet 2010; 282: 659-664

15 Walker JL, Piedmonte MR, Spirtos NM et al. Recurrence and survival after random assignment to laparoscopy versus laparotomy for comprehensive surgical staging of uterine cancer: Gynecologic Oncology Group LAP2 Study. J Clin Oncol 2012; 30: 695-700

16 Nemani D, Mitra N, Guo M et al. Assessing the effects of lymphadenectomy and radiation therapy in patients with uterine carcinosarcoma: a SEER analysis. Gynecol Oncol 2008; 111: 82-88

17 Sagae S, Yamashita K, Ishioka S et al. Preoperative diagnosis and treatment results in 106 patients with uterine sarcoma in Hokkaido, Japan. Oncology 2004; 67: 33-39

18 Schiavone MB, Zivanovic O, Zhou Q et al. Survival of patients with uterine carcinosarcoma undergoing sentinel lymph node mapping. Ann Surg Oncol 2016; 23: 196-202

19 Temkin SM, Hellmann M, Lee YC et al. Early-stage carcinosarcoma of the uterus: the significance of lymph node count. Int J Gynecol Cancer 2007; 17: 215-219

20 Galaal K, van der Heijden E, Godfrey K et al. Adjuvant radiotherapy and/ or chemotherapy after surgery for uterine carcinosarcoma. Cochrane Database Syst Rev 2013; 2: CD006812

21 Online: https://clinicaltrials.gov/ct2/show/NCT00954174; last access: 24.08.2015 
22 Aghajanian C, Sill MW, Secord AA et al. Iniparib plus paclitaxel and carboplatin as initial treatment of advanced or recurrent uterine carcinosarcoma: a Gynecologic Oncology Group Study. Gynecol Oncol 2012; 126: 424-427

23 Nimeiri HS, Oza AM, Morgan RJ et al. A phase II study of sorafenib in advanced uterine carcinoma/carcinosarcoma: a trial of the Chicago, $\mathrm{PMH}$, and California Phase II Consortia. Gynecol Oncol 2010; 117: 37-40

24 Huh WK, Sill MW, Darcy KM et al. Efficacy and safety of imatinib mesylate (Gleevec) and immunohistochemical expression of c-Kit and PDGFR-beta in a Gynecologic Oncology Group Phase Il Trial in women with recurrent or persistent carcinosarcomas of the uterus. Gynecol Oncol 2010; 117: 248-254

25 Mackay HJ, Buckanovich RJ, Hirte $H$ et al. A phase II study single agent of aflibercept (VEGF Trap) in patients with recurrent or metastatic gynecologic carcinosarcomas and uterine leiomyosarcoma. A trial of the Princess Margaret Hospital, Chicago and California Cancer Phase II Consortia. Gynecol Oncol 2012; 125: 136-140

26 Campos SM, Brady WE, Moxley KM et al. A phase II evaluation of pazopanib in the treatment of recurrent or persistent carcinosarcoma of the uterus: a gynecologic oncology group study. Gynecol Oncol 2014; 133 : 537-541
27 Colombo N, McMeekin DS, Schwartz PE et al. Ridaforolimus as a single agent in advanced endometrial cancer: results of a single-arm, phase 2 trial. Br J Cancer 2013; 108: 1021-1026

28 Salazar OM, Bonfiglio TA, Patten SF et al. Uterine sarcomas: analysis of failures with special emphasis on the use of adjuvant radiation therapy. Cancer 1978; 42: 1161-1170

29 Chi DS, Mychalczak B, Saigo PE et al. The role of whole-pelvic irradiation in the treatment of early-stage uterine carcinosarcoma. Gynecol Oncol 1997; 65: 493-498

30 Wright JD, Seshan VE, Shah $M$ et al. The role of radiation in improving survival for early-stage carcinosarcoma and leiomyosarcoma. Am J Obstet Gynecol 2008; 199: 536.e1-536.e8

31 Reed NS, Mangioni C, Malmström H et al.; European Organisation for Research and Treatment of Cancer Gynaecological Cancer Group. Phase III randomised study to evaluate the role of adjuvant pelvic radiotherapy in the treatment of uterine sarcomas stages I and II: an European Organisation for Research and Treatment of Cancer Gynaecological Cancer Group Study (protocol 55874). Eur J Cancer 2008; 44: 808-818 